\title{
Trombo auricular atrapado en el foramen oval: Complicación infrecuente en el tromboembolismo pulmonar
}

\author{
Ernesto Cairoli ${ }^{1,2}$, Carlos Codina ${ }^{3}$, Leandro Cura4, \\ Andrea Pino ${ }^{1}$, Juan Alonso ${ }^{1}$. \\ Atrial thrombus entrapped in a patent
foramen oval. Report of one case
}

We report a 63 year-old female with a pulmonary embolism in whom echocardiography revealed the presence of right heart thrombus. A section of this thrombus was entrapped in a patent foramen oval and floating in both atria. This rare situation, named impending paradoxical embolism, prompted us to perform a surgical intervention, removing the thrombus and repairing the foramen ovale (Rev Méd Chile 2008; 136: 753-6).

(Key words: Echocardiography; Foramen ovale; Pulmonary embolism)

\begin{abstract}
Recibido el 17 de julio, 2007. Aceptado el 1 de octubre, 2007.
${ }^{1}$ Departamento de Medicina, Clínica Médica "C", Prof. Dra. A. Belloso, ${ }^{2}$ Departamento Básico de Medicina, ${ }^{3}$ Departamento de Cardiología, ${ }^{4}$ Departamento de Cirugía Cardíaca, Hospital de Clínicas, Facultad de Medicina, Universidad de la República, Montevideo, Uruguay.
\end{abstract}

L

a presencia de trombos en cavidades cardíacas derechas (TCCD) en pacientes con tromboembolismo pulmonar (TEP) es un hallazgo asociado a mal pronóstico, siendo la ecocardiografía el método de estudio de elección ${ }^{1}$. La concomitancia de esta situación con la presencia de un foramen oval permeable, le otorga mayor severidad a la situación, dado el riesgo de producción de embolias paradojales hacia la circulación arterial sistémica. La actitud terapéutica en los casos de TEP con TCCD es un tema de debate, asociando un mayor nivel de complejidad frente a la inminencia de una embolia paradojal, donde las decisiones terapéuticas son aún más controvertidas. Presenta-

Correspondencia a: Dr. Juan Alonso. Hospital de Clínicas, Médica "C". Tele-fax 4878702. E mail: jaalonsobao@gmail.com mos el caso de una paciente en quien se diagnosticó un TEP evidenciando en el ecocardiograma TCCD, uno de los cuales se extendía desde la aurícula derecha hacia la izquierda a través del foramen oval permeable, quedando atrapado a ese nivel.

Caso clínico. Mujer de 63 años, con antecedentes de hipertensión arterial, que 20 días después de la extracción parcial de un meningioma maligno intracraneano (clase II O.M.S.) consultó por disnea de reposo de dos días de evolución, asociando dolor de miembros inferiores a predominio izquierdo. En la exploración física inicial la paciente se presentaba sin alteraciones en la conciencia, con presión arterial de 150/90 mmHg, frecuencia cardíaca de 120 latidos/min, polipnea de 24 respiraciones/min y estertores crepitantes en la base pulmonar derecha. A la auscultación cardíaca 
presentaba un ritmo regular con ruidos cardíacos sin alteraciones y sin soplos. No presentaba edemas ni signos de tromboflebitis en miembros inferiores. La presencia de factores de riesgo trombóticos (postoperatorio, reposo, enfermedad neoplásica) junto al cuadro clínico de polipnea, taquicardia y estertores pulmonares unilaterales en ausencia de fiebre, llevó al planteo diagnóstico de trombosis venosa profunda (TVP) de miembro inferior con TEP. La gasometría arterial evidenció hipoxemia. La radiografía de tórax no mostró alteraciones. El electrocardiograma presentaba una taquicardia sinusal. El eco-Doppler de miembros inferiores detectó una TVP fémoro-poplítea bilateral. Se realizó una gammagrafía pulmonar que mostró defectos de perfusión segmentarios a nivel de lóbulo inferior derecho, no concordantes con los hallazgos observados en la ventilación. Se inició tratamiento con heparina sódica intravenosa continuando con heparina de bajo peso molecular. Pasados siete días del inicio del tratamiento, se realizó un ecocardiograma transtorácico con el objetivo de valorar el desarrollo de hipertensión pulmonar luego del TEP. El mismo evidenció una masa hiperecogénica móvil a nivel de la aurícula derecha, por lo que se procedió a realizar un ecocardiograma transesofágico. Éste detectó un ventrículo izquierdo de dimensiones normales, con una fracción de eyección de $60 \%$. Mostró un ventrículo derecho levemente dilatado y a nivel de la aurícula derecha la presencia de una imagen hiperecogénica móvil, de aspecto serpenteante, de $9 \mathrm{~mm}$ de espesor en su sector más ancho, prolapsando a través de la válvula tricuspídea e invadiendo el ventrículo derecho, generando insuficiencia tricuspídea, pudiendo estimar la presión arterial pulmonar sistólica en $35 \mathrm{mmHg}$. Desde la aurícula derecha este trombo se extendía a través del foramen oval hacia la aurícula izquierda, manteniendo su movilidad pero quedando atrapado a ese nivel, ocupando parte de la aurícula izquierda e invadiendo el ventrículo izquierdo (Figura 1). Se observaron también trombos no móviles a nivel de la arteria pulmonar izquierda en su rama izquierda. Con la paciente clínicamente estable, frente a estos hallazgos, se inició heparina sódica intravenosa y se realizó una angiotomografía para evaluar la extensión de los trombos en el árbol vascular, detectando defectos en el relleno a nivel de ambas arterias pulmonares. Mediante cirugía cardíaca con circulación extracorpórea se extrajeron los trombos arteriales y cardíacos, realizando el cierre quirúrgico mediante sutura directa del foramen oval y colocando un filtro a nivel de la vena cava inferior. Se reinstaló la anticoagulación con heparina, transcurriendo el postoperatorio sin complicaciones, iniciándose posteriormente dicumarínicos hasta

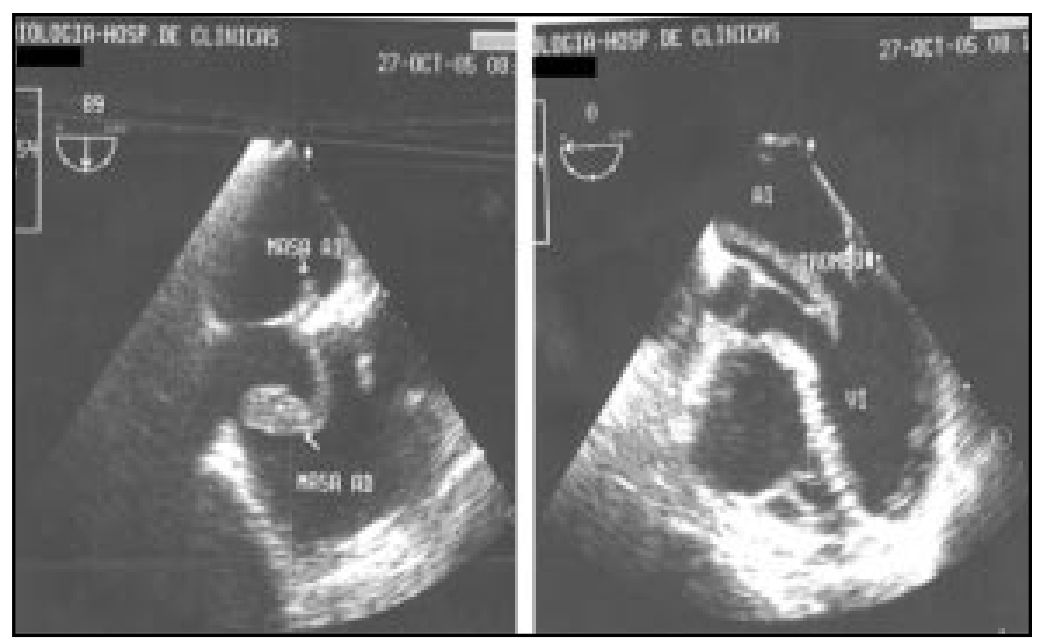

Figura 1. Ecocardiograma transesofágico donde se observa trombo de aspecto serpenteante a nivel de la aurícula derecha, extendiéndose hacia la aurícula izquierda, quedando atrapado a nivel del foramen oval (a izquierda). Se observa el mismo trombo recorriendo la aurícula izquierda e invadiendo el ventrículo izquierdo (a derecha). 
llegar a un INR de 2,5. Luego del alta, los estudios posteriores no demostraron el desarrollo de hipertensión arterial pulmonar, continuando de manera ambulatoria la radioterapia holocraneana para el tratamiento del meningioma.

\section{DisCUSIÓN}

Las complicaciones trombóticas postoperatorias luego de la neurocirugía aparecen con elevada incidencia, especialmente los casos de tumores malignos $^{2}$. La realización del ecocardiograma permitió detectar TCCD cambiando la orientación pronóstica y terapéutica de la paciente. Las posibles alteraciones detectables mediante ecocardiografía durante el TEP son la dilatación o disfunción ventricular derecha, movimiento septal paradojal, signos indirectos de hipertensión pulmonar, visualización directa del trombo sobre arterias pulmonares, TCCD y presencia del foramen oval permeable, entre otros hallazgos ${ }^{1}$. Esto la convierte en una herramienta diagnóstica complementaria de gran valor, siendo necesarios futuros ensayos clínicos para esclarecer las utilidades y limitaciones del ecocardiograma en el manejo del TEP ${ }^{1}$. La detección de TCCD mediante ecocardiografía es un evento poco frecuente, asociado casi en $100 \%$ de los casos a TEP3, pudiendo detectarse en $4 \%$ a $18 \%$ de los casos de TEP confirmado ${ }^{4}$. Existen diferentes tipos de trombo a nivel auricular de acuerdo a su morfología en la ecocardiografía. Un tipo de trombo es aquel extremadamente móvil, con forma de serpiente, capaz de prolapsar al ventrículo derecho y asociado a trombosis venosas profundas (características concordantes con la paciente presentada). El otro tipo de trombo es aquél de menor movilidad, adherido a las paredes cardíacas (trombo mural), similar a los encontrados en la aurícula izquierda y asociado a enfermedades cardíacas que desarrollan éstasis sanguíneo ${ }^{3}$. Sumado a esto debemos considerar que el hallazgo de TCCD durante el TEP ha sido asociado a una elevada mortalidad en las primeras $24 \mathrm{~h}$ de internación, llegando en algunas series a $45 \%$ de los $\operatorname{casos}^{4,5}$. En el caso presentado, se detectó la presencia de trombos a nivel de la arteria pulmonar y dentro de las cavidades cardíacas, observando la extensión de un trombo desde la aurícula derecha hacia la aurícula izquierda, pasando a través del foramen oval permeable, quedando atrapado a ese nivel, constituyendo una embolia paradojal inminente. $\mathrm{El}$ análisis de 38 pacientes consecutivos, en quienes fue diagnosticado un TEP con presencia de TCCD, encontró que sólo en 4 casos el trombo estaba atrapado a nivel del foramen oval ${ }^{5}$.

Una revisión reciente analizó 43 casos publicados de pacientes con diagnóstico de trombo atrapado a nivel del foramen oval, encontrando que $53 \%$ presentaron TEP, $40 \%$ la concomitancia TEP y embolia paradojal (hacia arterias cerebrales $50 \%$ y hacia miembros 30\%) y $7 \%$ presentó embolia paradojal únicamente ${ }^{6}$. Debe considerarse que en adultos sin cardiopatía, la presencia del foramen oval permeable tiene una incidencia de $27 \%{ }^{7}$, estando favorecida su apertura en las situaciones (como el TEP) donde la presión auricular derecha excede la presión auricular izquierda, posibilitando el desarrollo de una embolia paradojal ${ }^{1}$.

La opción terapéutica a utilizar en los casos de TCCD y TEP, es tema de controversia. Se ha comunicado que la mortalidad en los tratados sólo con heparina, cirugía o fibrinolíticos fue de $28 \%$, $23 \%$ y $11 \%$, respectivamente ${ }^{3}$. Si bien el tratamiento con fibrinolíticos parecería ser el conveniente, serán necesarios ensayos prospectivos, con selección aleatoria para determinar cuál es el tratamiento óptimo en estos casos $^{3}$. En el tratamiento de los casos de TEP con un trombo atrapado a nivel de foramen oval, los datos obtenidos proceden del análisis retrospectivo de casos aislados, reportándose como posibilidades terapéuticas el uso de anticoagulantes, fibrinolíticos y cirugía, teniendo una mortalidad de $36 \%, 16 \%$ y $13 \%$, respectivamente ${ }^{6}$. Se ha propuesto que la utilización de fibrinolíticos podnía asociarse a la fragmentación del trombo con la consecutiva embolización sistémica, por lo que la cirugía podnía ser la opción terapéutica más adecuada 6 . En el caso analizado, la presencia de una neurocirugía reciente contraindicaba el uso de fibrinolíticos, y al mismo tiempo, la presencia del trombo atrapado en el foramen oval marcaba la necesidad de realizar una cirugía cardíaca de emergencia. En el mismo acto quirúrgico se procedió a realizar las embolectomías, el cierre del foramen oval y la colocación de un filtro en la vena cava. El tratamiento constituye una auténtica emergencia, donde las opciones terapéuticas dependerán de la estabilidad hemodinámica del 
paciente, la presencia de contraindicaciones para las opciones terapéuticas y de la disponibilidad de dichas opciones en el centro hospitalario. En los casos de trombos atrapados a nivel del foramen oval, los resultados disponibles corresponden a la compilación de casos heterogéneos resultado de reportes individuales, por lo que al momento no se disponen de datos estadísticamente significativos que permitan comparar las diferentes modalidades terapéuticas, si bien la cirugía representa una de las opciones con menor mortalidad ${ }^{6,8}$.

\section{REFERENCIAS}

1. GOLDHABER S. Echocardiography in the management of pulmonary embolism. Ann Intern Med 2002; 136: 691-700.

2. Geerts W, Pineo G, Heit J, Bergqvist D, Lassen M, Colwell $C$ et al. Prevention of venous thromboembolism. Chest 2004; 126: 338s-400s.

3. Rose P, Punjabi N, Pearse D. Treatment of right heart thromboemboli. Chest 2002; 121: 806-14.

4. Torbicki A, Galié N, Covezzol B, Rossi E, De Rosa $\mathrm{M}$, GoLDHABER S. Right heart thrombi in pulmonary embolism. J Am Coll Cadiol 2003; 41: 2245-51.

5. Chartier L, Béra J, Delomez M, Asseman P, Beregi JP, Bauchart JJ et al. Free-Floating thrombi in the
En conclusión, pensamos que la realización de la ecocardiografía posibilitó la identificación de una situación de mayor riesgo como fue la detección de TCCD y que la rápida intervención evitó la reiteración del TEP y la embolización hacia la circulación sistémica del trombo atrapado a nivel del foramen oval. La opción terapéutica de elección en los casos de TEP con TCCD aún resta por conocerse, siendo necesarios el desarrollo de estudios prospectivos que lo analicen.

right heart. Diagnosis, management, and prognostic indexes in 38 consecutive patients. Circulation 1999; 99: 2779-83.

6. Aboyans V, Lacroix P, Ostyn E, Cornu E, Laskar M. Diagnosis and management of entrapped embolus through a patent foramen ovale. Eur J CardioThorac Surg 1998; 14: 624-8.

7. Hagen P, Scholz D, Edwards W. Incidence and size of patent foramen ovale during the first 10 decades of life: an autopsy study of 965 normal hearts. Mayo Clin Proc 1984; 59: 12-20.

8. Meacham R, Headley S, Bronze M, Lewis J, Rester M. Impending paradoxical embolism. Arch Intern Med 1998; 158: 438-48. 